Supplementary Materials for

\title{
Highly Enantioselective Phenylacetylene Additions to Ketones Catalyzed by (S)-BINOL-Ti Complex
}

Yi-feng Zhou, Rui Wang, ${ }^{*}$ Zhao-qing Xu, Wen-jin Yan, Lei Liu, Yong-feng Kang, Zhi-jian Han

Analysis of the optically Active Tertiary Propargylic Alcohols.

2-(4-Chloro-phenyl)-4-phenyl-but-3-yn-2-ol: 73\% yield isolated after $48 \mathrm{~h}$ reaction in Step 3. 89\% ee determined by HPLC analysis (Chiralcel OD column, 5\% IPA in hexane, $241.5 \mathrm{~nm})$. Retention time: $\mathrm{t}_{\text {major }}=8.78$ and $\mathrm{t}_{\text {minor }}=10.54 \mathrm{~min} .{ }^{1} \mathrm{H}$ NMR $(200$ $\left.\mathrm{MHz} \mathrm{CDCl}_{3}\right) \delta$ 7.68-7.31 (m,2H), 7.49-7.44 (m, 2H), 7.35-7.31 (m, 5H), 2.60 (brs, $1 \mathrm{H}), 1.84(\mathrm{~s}, 3 \mathrm{H}) .{ }^{13} \mathrm{C} \mathrm{NMR}\left(50 \mathrm{MHz}, \mathrm{CDCl}_{3}\right) \delta 144.2,133.5,131.7,128.6,128.4$, $128.3,126.5,122.3,91.9,85.2,69.9,33$.

4-Phenyl-2-m-tolyl-but-3-yn-2-ol: $66 \%$ yield isolated after $48 \mathrm{~h}$ reaction in Step 3. 90\% ee determined by HPLC analysis (Chiralcel OD column, 5\% IPA in hexane, $241.5 \mathrm{~nm})$. Retention time: $\mathrm{t}_{\text {major }}=9.19$ and $\mathrm{t}_{\text {minor }}=10.91 \mathrm{~min} .{ }^{1} \mathrm{H}$ NMR $(200$ $\left.\mathrm{MHz}, \mathrm{CDCl}_{3}\right) \delta$ 7.54-7.45 (m, 4H), 7.32-7.23 (m, 4H), $7.12(\mathrm{~d}, 1 \mathrm{H}), 2.38(\mathrm{~s}, 3 \mathrm{H})$, $1.86(\mathrm{~s}, 3 \mathrm{H}) .{ }^{13} \mathrm{C} \mathrm{NMR}\left(50 \mathrm{MHz}, \mathrm{CDCl}_{3}\right) \delta 145.6,138.0,131.7,128.4,128.2$, 125.6, 122.6, 122.1, 119.8, 92.7, 84.7, 70.3, 33.2, 21.5.

2,4-Diphenyl-but-3-yn-2-ol: $67 \%$ yield isolated after $48 \mathrm{~h}$ reaction in Step 3. $85 \%$ ee determined by HPLC analysis (Chiralcel OD column, 5\% IPA in hexane, $241.5 \mathrm{~nm})$. Retention time: $\mathrm{t}_{\text {major }}=9.15$ and $\mathrm{t}_{\text {minor }}=11.02 \mathrm{~min}$. ${ }^{1} \mathrm{H}$ NMR $(200$ $\left.\mathrm{MHz}, \mathrm{CDCl}_{3}\right) \delta 7.74(\mathrm{~d}, 2 \mathrm{H}), 7.47-7.26(\mathrm{~m}, 8 \mathrm{H}), 2.5(\mathrm{~s}, 1 \mathrm{H}), 1.87(\mathrm{~s}, 3 \mathrm{H})$.

2-(3-Bromo-phenyl)-4-phenyl-but-3-yn-2-ol: $68 \%$ yield isolated after $48 \mathrm{~h}$ reaction in Step 3. 86\% ee determined by HPLC analysis (Chiralcel OD column, 5\% IPA in hexane, $241.5 \mathrm{~nm})$. Retention time: $\mathrm{t}_{\text {major }}=9.59$ and $\mathrm{t}_{\text {minor }}=12.08 \mathrm{~min} .{ }^{1} \mathrm{H}$ NMR $(200$ $\left.\mathrm{MHz}, \mathrm{CDCl}_{3}\right) \delta 7.88(\mathrm{~m}, 3 \mathrm{H}), 7.66-7.20(\mathrm{~m}, 8 \mathrm{H}), 2.55$ (brs, $\left.1 \mathrm{H}\right), 1.85$ (s, 3H). ${ }^{13} \mathrm{C}$ NMR $\left(50 \mathrm{MHz}, \mathrm{CDCl}_{3}\right) \delta 147.9,131.7,130.8,129.9,128.6,128.3,123.7,122.4$, $122.2,92.3,85.0,69.9,33.4$.

2-(3-Methoxy-phenyl)-4-phenyl-but-3-yn-2-ol: $81 \%$ yield isolated after $48 \mathrm{~h}$ reaction in Step 3. 92\% ee determined by HPLC analysis (Chiralcel OD column, 5\% IPA in hexane, $241.5 \mathrm{~nm}$ ). Retention time: $\mathrm{t}_{\text {major }}=12.67$ and $\mathrm{t}_{\text {minor }}=15.69 \mathrm{~min}$. 
3-Methyl-1,5-diphenyl-pent-1-en-4-yn-3-ol: $88 \%$ yield isolated after $18 \mathrm{~h}$ reaction in Step 3.73\% ee determined by HPLC analysis (Chiralcel OD column, 5\% IPA in hexane, $254 \mathrm{~nm}$ ). Retention time: $\mathrm{t}_{\text {major }}=14.42$ and $\mathrm{t}_{\text {minor }}=28.25 \mathrm{~min}$.

2-Naphthalen-2-yl-4-phenyl-but-3-yn-2-ol: $72 \%$ yield isolated after $48 \mathrm{~h}$ reaction in Step 3. 85\% ee determined by HPLC analysis (Chiralcel OD column, 5\% IPA in hexane, $225 \mathrm{~nm})$. Retention time: $\mathrm{t}_{\text {major }}=13.59$ and $\mathrm{t}_{\text {minor }}=18.30 \mathrm{~min}$. ${ }^{1} \mathrm{H}$ NMR (200 $\left.\mathrm{MHz} \mathrm{CDCl}_{3}\right) \delta 8.18(\mathrm{~s}, 1 \mathrm{H}), 7.87-7.77(\mathrm{~m}, 4 \mathrm{H}), 7.52-7.45(\mathrm{~m}, 4 \mathrm{H}), 7.34-7.30(\mathrm{~m}$, $3 \mathrm{H}), 2.68$ (brs, $1 \mathrm{H}), 1.95$ (s, 3H). ${ }^{13} \mathrm{C} \mathrm{NMR}\left(50 \mathrm{MHz}, \mathrm{CDCl}_{3}\right) \delta 142.9,133.0,132.9$, $131.8,128.5,128.3,128.2,126.2,126.1,122.6,92.5,85.1,70.5,33.1$.

2-(2-Fluoro-phenyl)-4-phenyl-but-3-yn-2-ol: $80 \%$ yield isolated after $48 \mathrm{~h}$ reaction in Step 3. 66\% ee determined by HPLC analysis (Chiralcel OD column, 5\% IPA in hexane, $241.5 \mathrm{~nm})$. Retention time: $\mathrm{t}_{\text {major }}=9.80$ and $\mathrm{t}_{\text {minor }}=12.18 \mathrm{~min} .{ }^{1} \mathrm{H}$ NMR $(200$ $\left.\mathrm{MHz}, \mathrm{CDCl}_{3}\right) \delta$ 7.78-.7.70 (m, 1H), 7..48-7.44 (m, 2H), 7.32-7.25 (m, 4H), 7.19-7.04 $(\mathrm{m}, 2 \mathrm{H}), 2.79$ (brs, $1 \mathrm{H}), 1.98(\mathrm{~s}, 3 \mathrm{H}) .{ }^{13} \mathrm{C}$ NMR $\left(50 \mathrm{MHz}, \mathrm{CDCl}_{3}\right) \delta 131.7,129.7$, $129.5,128.5,128.2,124.0,123.9,116.6,116.1,92.6,84.1,68.1,30.6$.

4-Phenyl-2-p-tolyl-but-3-yn-2-ol: $64 \%$ yield isolated after $48 \mathrm{~h}$ reaction in Step 3. 87\% ee determined by HPLC analysis (Chiralcel OD column, 5\% IPA in hexane, $241.5 \mathrm{~nm})$. Retention time: $\mathrm{t}_{\text {major }}=8.86$ and $\mathrm{t}_{\text {minor }}=11.71 \mathrm{~min} .{ }^{1} \mathrm{H}$ NMR $(200$ $\left.\mathrm{MHz}, \mathrm{CDCl}_{3}\right) \delta 7.60(\mathrm{~d}, 2 \mathrm{H}), 7.50-7.45(\mathrm{~m}, 2 \mathrm{H}), 7.33-7.29(\mathrm{~m}, 3 \mathrm{H}), 7.18(\mathrm{~d}, 2 \mathrm{H}), 2.50$ (s, 1H), $2.34(\mathrm{~s}, 3 \mathrm{H}), 1.86(\mathrm{~s}, 3 \mathrm{H}) .{ }^{13} \mathrm{C} \mathrm{NMR}\left(50 \mathrm{MHz}, \mathrm{CDCl}_{3}\right) \delta 142.8,137.4,131.7$, $129.0,128.4,128.3,124.9,119.2,92.1,85.4,70.2,33.2,21.0$. 


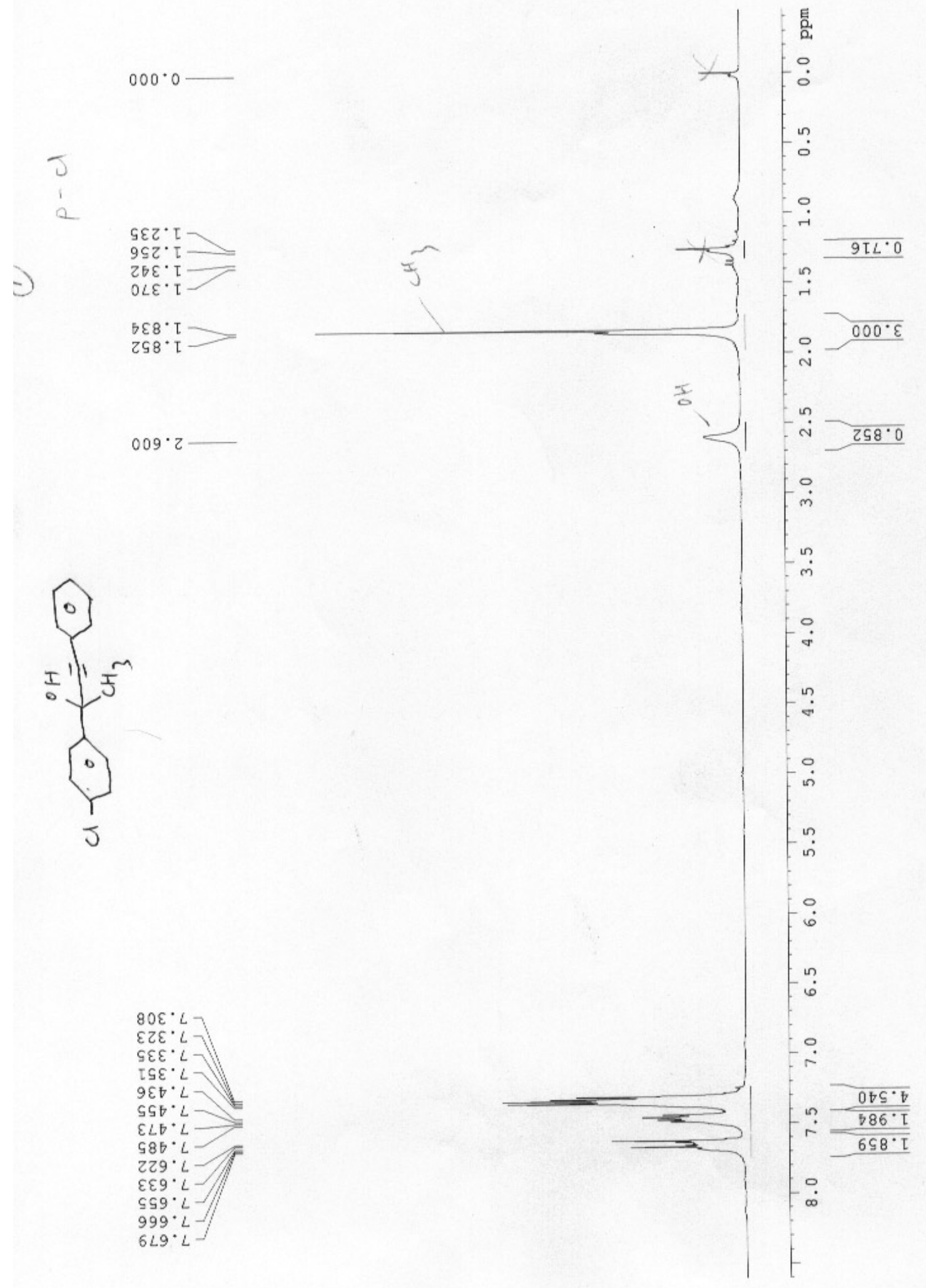




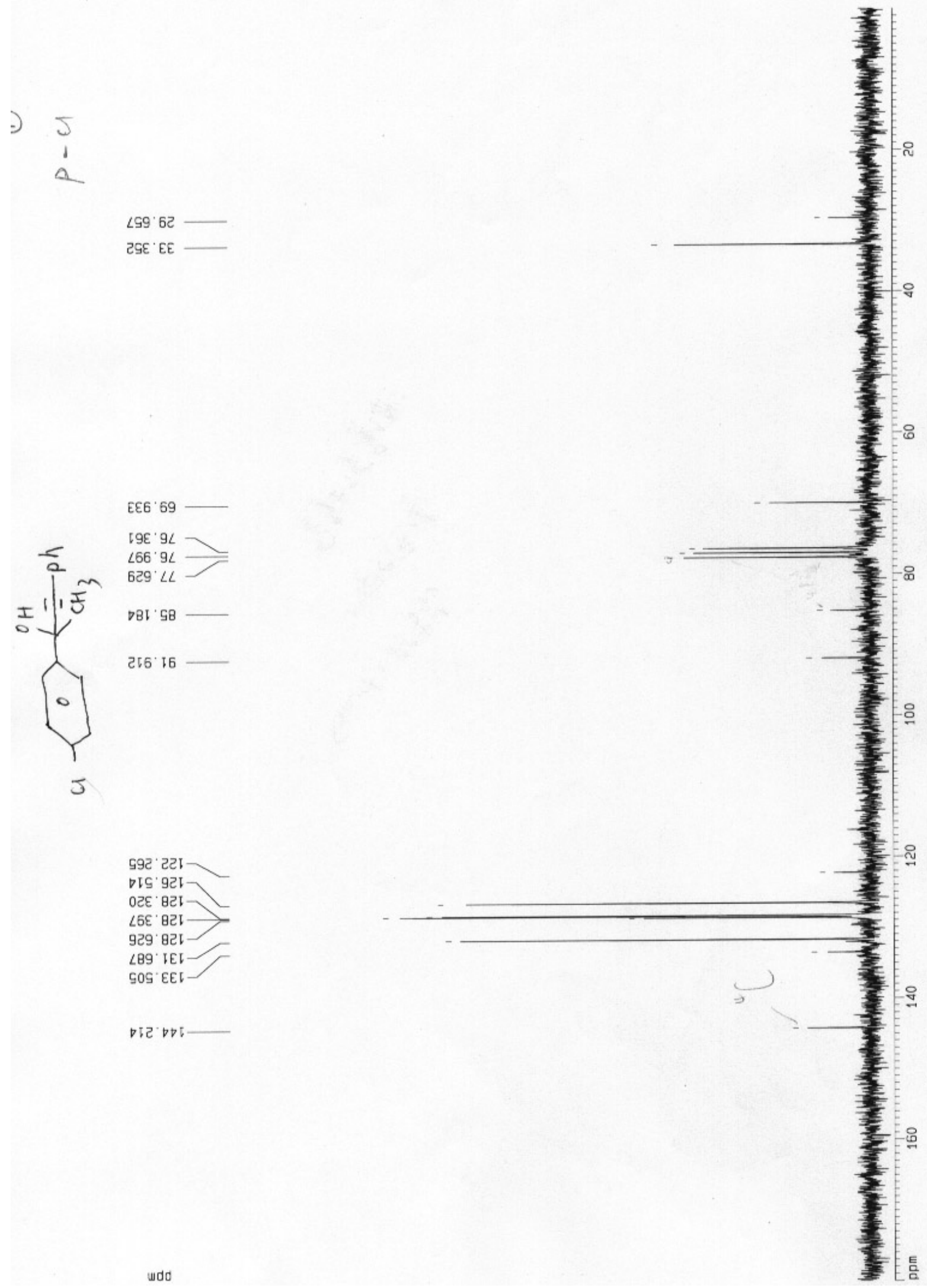




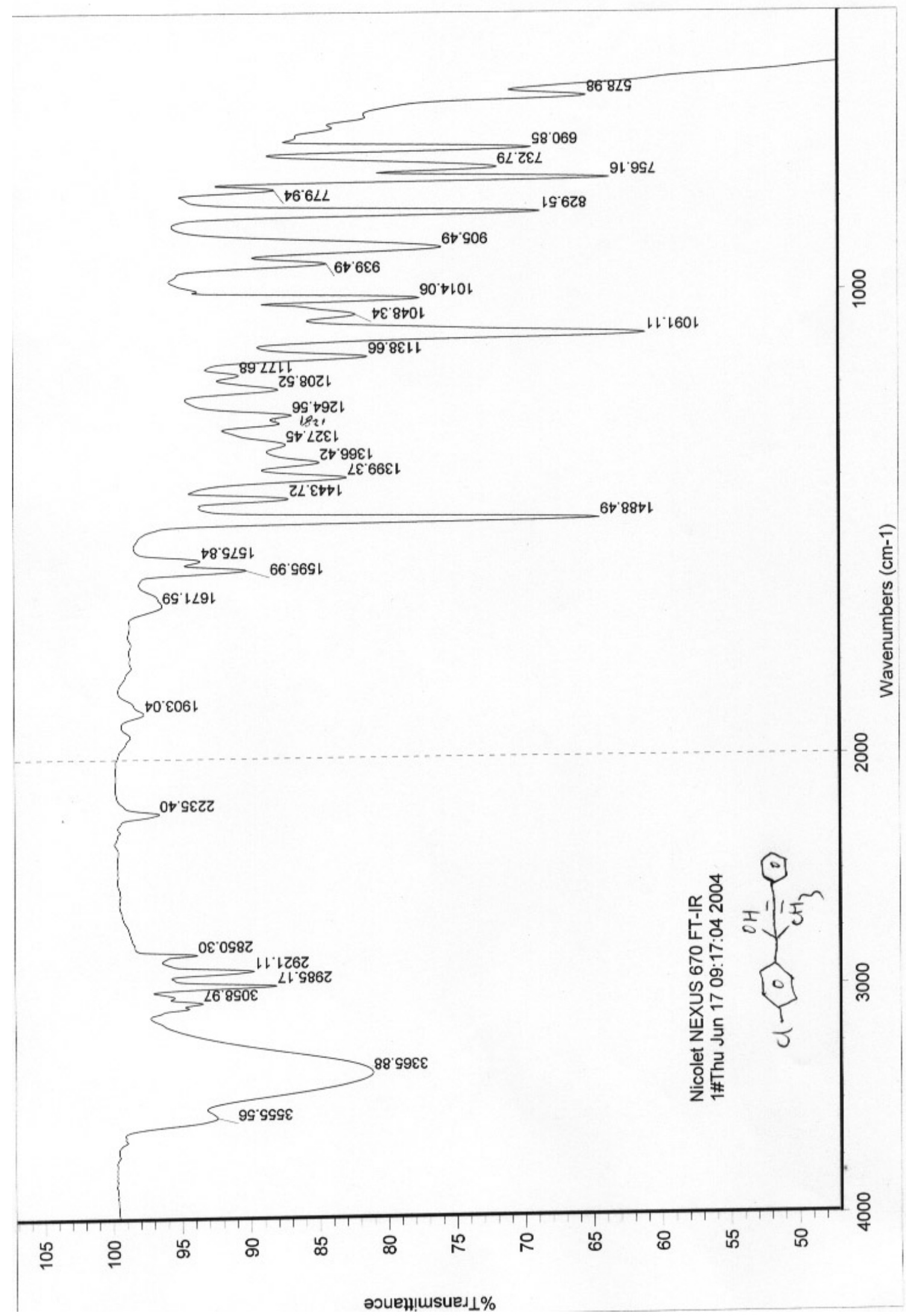




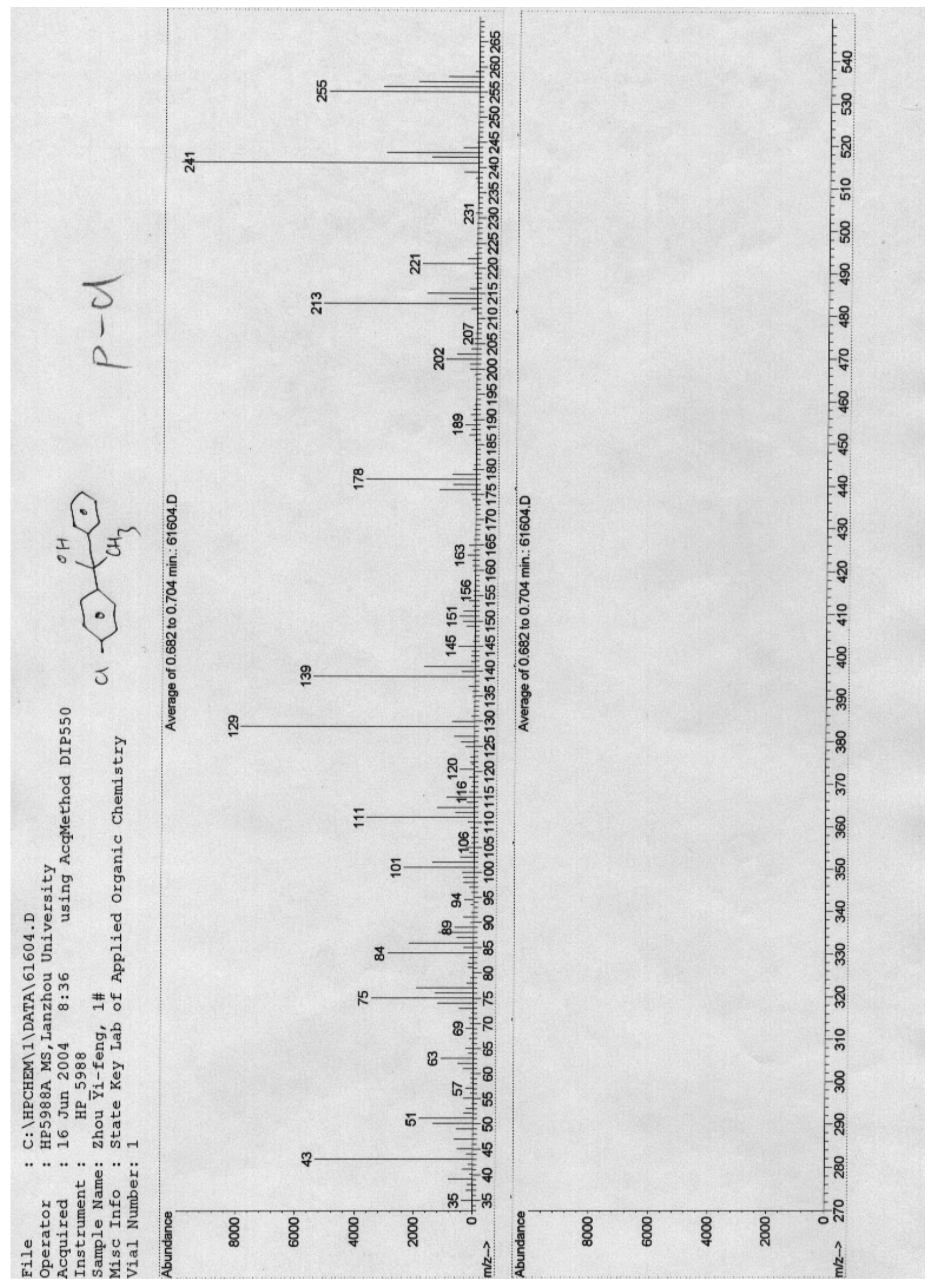




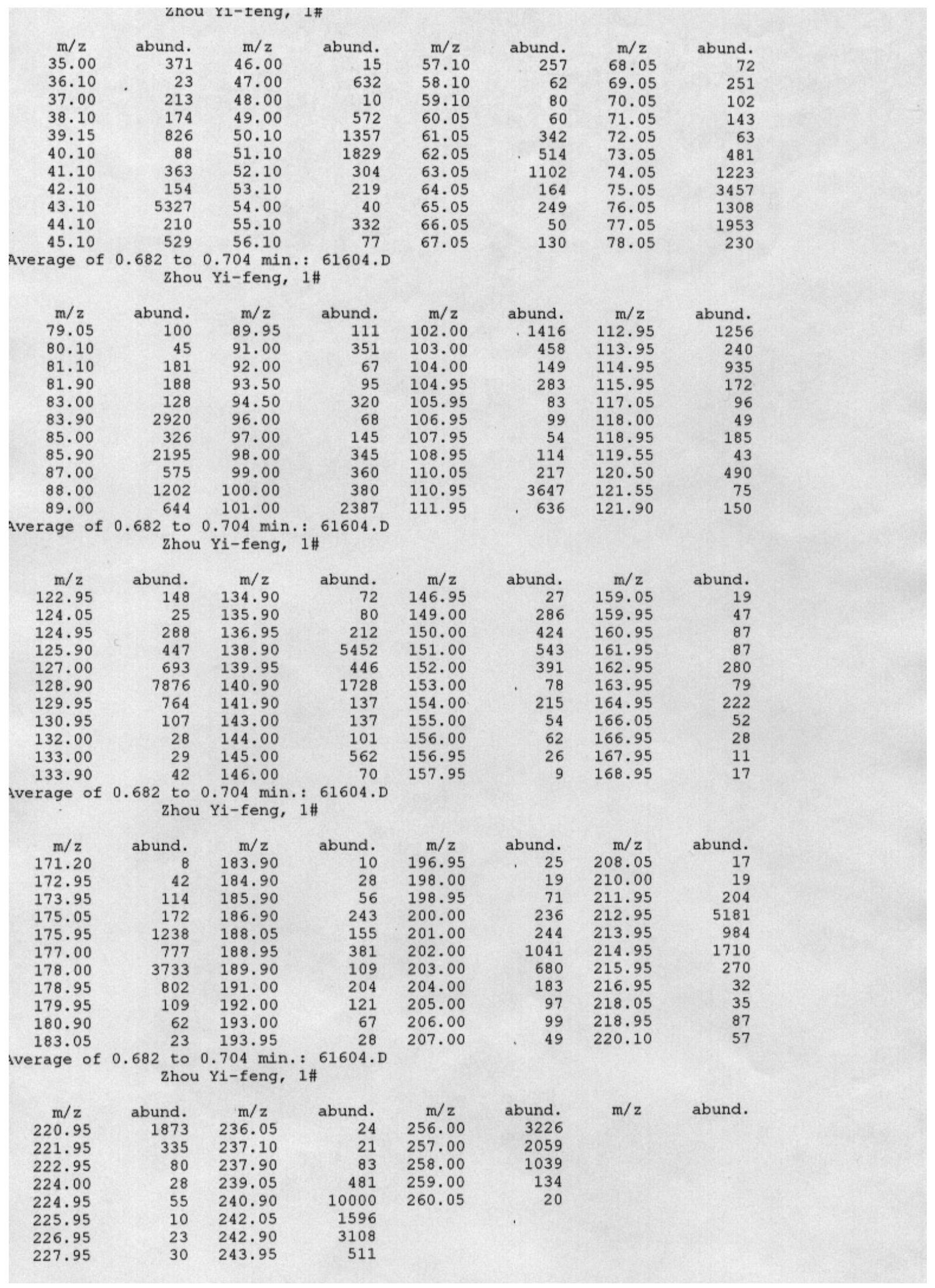




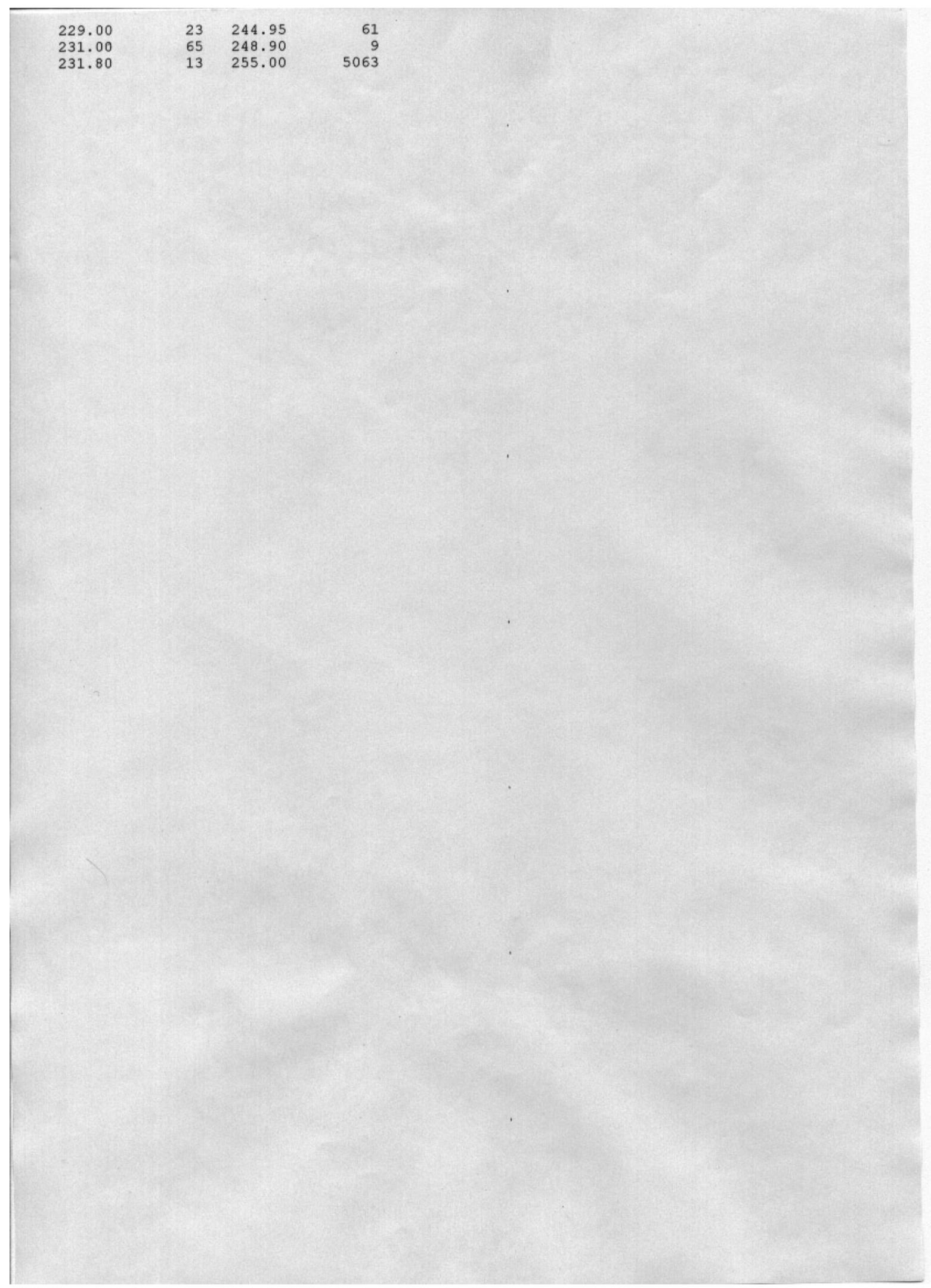

